\title{
Random Network Transmission and Countermeasures in Containing Global Spread of COVID-19-Alike Pandemic: A Hybrid Modelling Approach
}

\author{
Yimin Zhou $\mathbb{D}^{1},{ }^{1}$ Jun Li ${ }^{D},{ }^{2,3}$ Lingjian Ye ${ }^{(D)},{ }^{1,4}$ Zuguo Chen ${ }^{(D)},{ }^{1}$ Qingsong Luo $\left(\mathbb{D},{ }^{1,4}\right.$ \\ Xiangdong $\mathrm{Wu}\left(\mathbb{D},{ }^{1,4}\right.$ and Haiyang $\mathrm{Ni} \mathbb{1}^{5}$ \\ ${ }^{1}$ Shenzhen Institutes of Advanced Technology, Chinese Academy of Sciences, Shenzhen 518055, Guangdong Province, China \\ ${ }^{2}$ School of International Relations, Sun Yat-sen University, Guangzhou, China \\ ${ }^{3}$ School of Management, Curtin University, Bentley, Australia \\ ${ }^{4}$ The University of Chinese Academy of Sciences, Beijing, China \\ ${ }^{5}$ Tianjin Academy of Traditional Chinese Medicine Affiliated Hospital, Tianjin 300120, China
}

Correspondence should be addressed to Yimin Zhou; ym.zhou@siat.ac.cn and Jun Li; jun.li@curtin.edu.au

Yimin Zhou, Jun Li, and Lingjian Ye contributed equally to this work.

Received 13 May 2020; Revised 15 September 2020; Accepted 20 October 2020; Published 10 November 2020

Academic Editor: Dan Selişteanu

Copyright (C) 2020 Yimin Zhou et al. This is an open access article distributed under the Creative Commons Attribution License, which permits unrestricted use, distribution, and reproduction in any medium, provided the original work is properly cited.

Since the outbreak of the novel coronavirus disease (COVID-19) at the beginning of December 2019, there have been more than 28.69 million cumulative confirmed cases worldwide as of $12^{\text {th }}$ September 2020, affecting over 200 countries and regions with more than 920,463 deaths. The COVID-19 pandemic has been sweeping worldwide with unexpected rapidity. In this paper, a hybrid modelling strategy based on tessellation structure- (TS-) configured SEIR model is adopted to estimate the scale of the pandemic spread. Building on the data pertaining to the global pandemic transmission over the last six months around the world, key impact factors in the transmission and control procedure have been analysed, including isolation rate, number of the infected cases before taking prevention measures, degree of contact scope, and medical level, so as to capture the fundamental factor influencing the pandemic. The quantitative evaluation allowed us to illustrate the magnitude of risks of pandemic and to recommend appropriate national health policy of prevention measures for effectively controlling both intra- and interregional pandemic spread. Our modelling results clearly indicate that the early-stage preventive measures are the most effective action to be taken to contain the pandemic spread of the highly contagious nature of the COVID-19.

\section{Introduction to the COVID-19 Pandemic Development}

Since the outbreak of novel coronavirus disease (COVID-19) at the beginning of December 2019, over 29.96 million people are infected with the disease, with 920,463 death cases around the world as of $12^{\text {th }}$ September 2020. There are 87 countries whose confirmed cases exceed 10,000, affecting over 200 countries in the world at amazing width and rapidity against expectation. The risk level of COVID-19 pandemic was raised to "very high" on $28^{\text {th }}$ February 2020 by the World Health Organization (WHO) since the global community is being rampaged by the disease [1]. The current distribution of COVID-19 pandemic cases around the world is shown in Figure 1 [2].

The COVID-19 virus has been proven as a pandemic at the global level $[4,5]$. As it is known, the rapid declination in the number of the new infection cases in China was ascribed to the strict quarantine measures (i.e., including lockdown of Wuhan and suspension of all outdoor activities and public entertainment facilities) and timely construction of makeshift hospitals to confine infected patients, while the situation in other countries, i.e., South Korea, Japan, Iran, Italy, and France, was deteriorating rapidly at the same time. The 


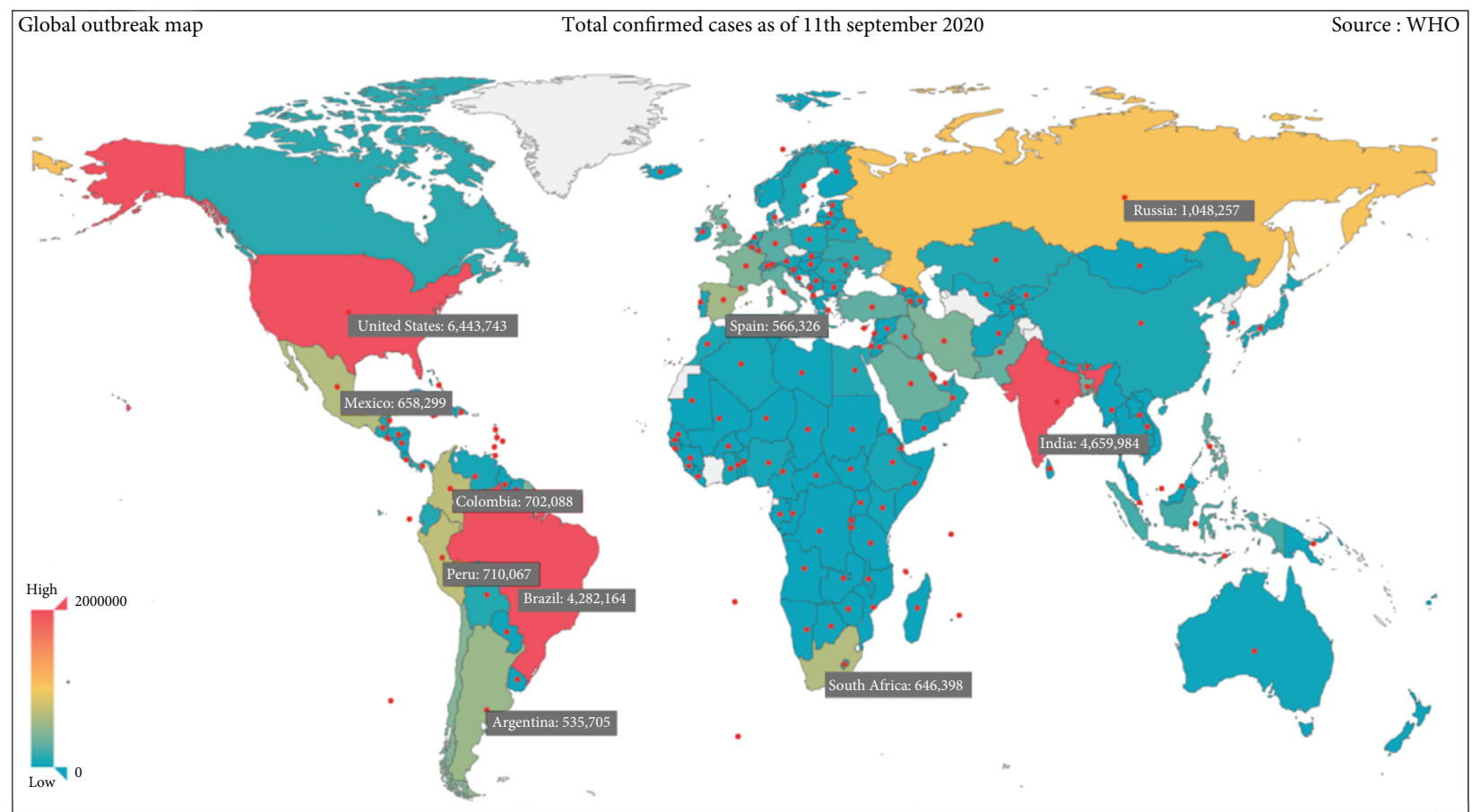

FIGURE 1: Distribution of global COVID-19 outbreak hotspots. As of 19:30, $12^{\text {th }}$ September 2020 , the global confirmed cases were $28,692,299$ and death cases 920,463. Over 1000 cases were confirmed in more than 15 countries, while 97570 cases were reported in India, 47855 cases in USA, and 43718 cases in Brazil (source: WHO [3]).

preventive measures taken by the government in dealing with the virus prevention and control are beneficial for understanding the transmission feature of the COVID-19 for the purpose of effective containment $[4,6]$.

The pandemic control experiences learned from China would be invaluable to other countries $[7,8]$. However, interventionist versus laissez-faire are the two different attitudes among the infected countries since the outbreak of COVID-19. The former is concerned with active inventions including radical confinement and quarantine measures, whereas the latter takes rather a sangfroid position, i.e., loose control or only providing suggestion in dealing with the virus transmission. For instance, Italy has taken similar strict prevention measures as in China, i.e., to close down the seriously infected regions and block the interpersonal spread at the early stage. Iranian government has also taken a series of intervention measures, e.g., closed public places, cancelled sports events, and suspended school nationwide. It should be noted that the pandemic prevention measures issued by the government would strictly be abided by the Iranian public. In contrast, only medical advice was provided by the government to the public in certain countries, without any further compulsory action taken to stop the community transmission at the early stage, allowing pandemic deteriorated rapidly.

Which measures are the appropriate ones to be taken by the governments have controversial debates, leading to various development directions of the highly contagious pandemic. In the meantime, the key factors influencing the COVID-19 transmission routes and infection rates should be investigated thoroughly to maximize the effectiveness of policy intervention with reasonable acceptable social cost to constraint the COVID-19 spread. Different modelling methods are applied to study the dynamics of the spread of infectious diseases such as susceptible-exposed-infectiousremoved (SEIR) model [9], stochastic transmission model [10], and neural network model. However, the contact network effect, i.e., interpersonal contact times and scope, has seldom been taken into the modelling consideration. Therefore, to which certain level of the prevention measure should be adopted remains inconclusive without reaching a unanimous recognition although the control measures from certain countries have been proved to be effective.

In order to bridge this knowledge gap, an improved SEIR (susceptible-exposed-infectious-recovered) model embedded with tessellation structures (TSs) is developed to estimate the transmission tendency of the COVID-19 considering the key parameters in the procedure under different scenarios. It allows the random behaviour of interpersonal contact to be considered as probabilistic network parametric impact along with other factors, including isolation rate, contact number during gathering at regularity, and the size of the initial infected cases, from which the effective containment strategies, i.e., contact tracing or isolation measures, can be discussed and recommended accordingly. Based on the pandemic situation dealt in China and other early outbreak countries, the results presented in the paper clearly indicate that proactive prevention measures and effective medical resources providence are the most important actions to be adopted under high risk of COVID19 , especially in religious gathering areas and densely inhabited districts. 
The remainder of the paper is organized as follows. Section 2 introduces the involved method with SEIR model combined with TS to form the virus transmission network. The key factors influencing the transmission speed and scope are analysed in Section 3. Discussion dealing with COVID19 locally and globally to determine which measures should be adopted in different scenarios is provided in Section 4 . Section 5 concludes the paper.

\section{The COVID-19 Transmission Modelling Strategy}

In this study, the standard SEIR (susceptible-exposed-infectious-recovered) model is coupled with a tessellation structure algorithm (TS) for describing each state in order to simulate the transmission of COVID-19 [11]. TSs are spatially and temporally discrete, abstract computational systems which can be used to describe nonlinear dynamics and composed of a set of homogenous and simple units. They evolve in parallel at discrete time steps considering the states of cells in their local neighbourhood, which can compute functions and emulate a universal computable problem, such as the coronavirus transmission pattern impacted by many stochastic factors [12]. In addition, the discrete random network characteristics of TS are particularly appropriate for investigating the effectiveness of the prevention and control strategy.

The developed TS-SEIR model has 7 states, such as $S, E, I$, $R$, Eq, $H$, and $D$, where the state setting of TS is designed based on four primary factors during COVID-19 control: (1) a 14-day quarantine period for an individual who comes from high-risk area or susceptible to be exposed by the infected; (2) individuals are highly suggested not going outside unless necessary; (3) individuals are restricted to participate in gatherings; and (4) patients should be admitted timely. In a typical mathematical model for an epidemic study, the number of the susceptible, latent, infectious, and removed individuals is represented as $S, E, I$, and $R$, respectively [13], while $\mathrm{Eq}, H$, and $D$ are the quarantined latent, quarantined infectious, and dead individuals, as illustrated in Figure 2.

There are total 14 rules to describe the state transmission: (1) the susceptible individuals become exposed and enter the latent period without quarantine after contact with the infected; (2) the susceptible individuals are quarantined and enter the latent period after contact with the infected; (3) the exposed individuals are quarantined; (4) the infected individuals are quarantined; (5) the exposed individuals without being in quarantine were infected after 14 days (average period of time); (6) the quarantined of the exposed individuals are confirmed infection and begin a quarantine of higher level; (7) the exposed individuals were infected and quarantined; (8) the infected individuals without quarantine recovered; (9) the quarantined of the exposed individuals are recovered without infection (small probability); (10) the quarantined of the infected individuals recovered; (11) the infected individuals without quarantine died; (12) the quarantined of the infected died; (13) the exposed individuals without quarantine infect the susceptible through contact; and (14) the infected individuals without quarantine infect the susceptible through contact.

It is assumed that the number of individuals in contact with other individuals in the unit time (one day) obeys a Poisson distribution, and then, the probability of $m$ individuals in contact with $j$ individuals per day is calculated as

$$
P_{j}(x)=\frac{\omega^{j}}{j !} e^{-\omega}\left(j=0,1,2, \ldots, D_{e_{m}}\right),
$$

where $D_{e_{m}}$ is the node degree of individual $m$ in the interpersonal contact network and the average number of each individual's contact with other people in one day is denoted as $\omega$. Thus, the probability of the $i^{\text {th }}$ individual being infected is

$$
p_{i}=\max _{1 \leq m \leq n}\left\{p_{i m}\right\},
$$

where $n$ is the total number of the individual nodes, and the infection probability $p_{i m}$ can be calculated by the following equation:

$$
p_{i m}=\frac{l_{i m} I_{x}}{D_{e_{m}}} \sum_{j=1}^{D_{e_{m}}} \frac{\omega^{j} e^{-\omega}}{(j-1) !} .
$$

It should be noted that there are small portions of people who have not been isolated in latent states but, with a larger node degree, would become more dangerous in an interpersonal contact network [14]. The developed TS-SEIR model can simulate the virus propagation, where each individual is treated as a cell evolving different states varied along with the time and space. The rules of the state variation are designed based on the characteristics of virus transmission. The implementation of programming the TS-SEIR model in our study follows the strategy as described below.

Formally, a TS is represented by a 4 -tuple $(Z, S, N, F) . Z$ represents the number of discrete regular grid of cells, i.e., the number of simulated population; $S$ represents the set of cell states; $N$ represents the neighbourhood; and $F$ represents the transition functions or transition rules. Here, it is set as $Z=1 \times 10000$, meaning one-dimensional TS. The unit for describing the transmission network having 7 states is written as

$$
S=\{S, E, I, R, \mathrm{Eq}, H, D\} .
$$

$N$ is an adjacency matrix of the unit with $10000 \times 10000$ dimensions. Then, the transition rules $F$ (see Figure 2) of TSSEIR model are described as Pseudocode 1.

Several parameters for describing the transmission degree are involved in the TS-SEIR model, which are listed in Table 1. $q_{1} / q_{2}$ is the isolation rate, where $q_{1}$ is the isolation rate in the latent period and $q_{2}$ is the isolation rate in the infection period. The $1^{\text {st }}$ group experiments are focused on the degree of the variation rate where the medical level index is set as 0.9455 based on the fact. For instance, $0.9 / 0.99$ is set due to the strict isolation measures taken in certain countries, and it is also taken in the following experiments. The $2^{\text {nd }}$ group experiments deal with the comparison of normal contact times and gathering contact times during virus 


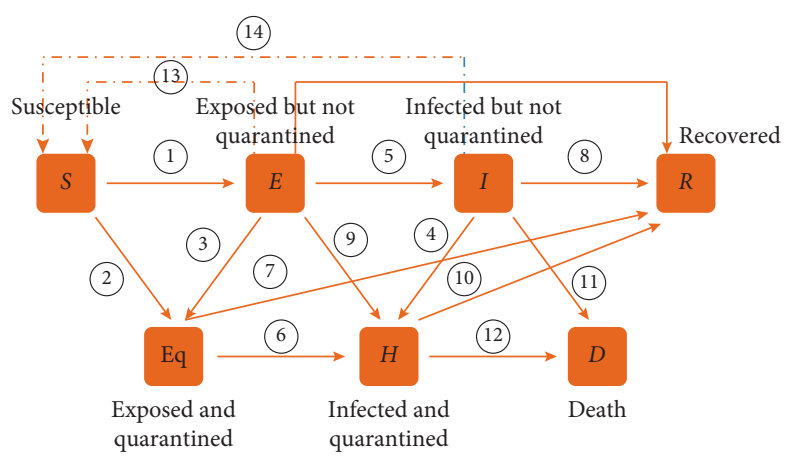

FIgURE 2: The epidemic transmission model.

\section{/*}

Parameters settings:

$q_{1} \longleftarrow 0.9 ; q_{2} \longleftarrow 0.99$;

normalContactTimes $\longleftarrow 6$;

gatheringContactTimes $\longleftarrow 80$;

initialInfectNum $\longleftarrow 100$;

medicalLevel $\longleftarrow 0.0413$;

*l

//Initialize the adjacency matrix according to the value of normalContactTimes and gatheringContactTimes

//Initialize the number of cell with states $E$ and $I$ according to the value of initialInfectNum

for $t \longleftarrow 1$ to simulatedTimeStep

do for $i \longleftarrow 1$ to populationNum

if $\operatorname{state}(t, i)=S \& \&$ anyNeighborOfCell $(i)=E$ or $I \& \&$ infectRate $\geq$ rand

then state $(t+1, i) \longleftarrow E$;

if state $(t, i)=E \& \&$ probTurnToInfected $\geq$ rand $\& \& q_{2} \geq$ rand then state $(t+1, i) \longleftarrow H$;

if state $(t, i)=E \& \&$ probTurnToInfected $\geq$ rand $\% \% q_{2}<r$ and then state $(t+1, i) \longleftarrow I$;

if state $(t, i)=S$ or $E \& \&$ anyNeighborOfCell $(i)=I$ or $H \& \&$ daysAfterContact $(i$, indexOfThisNeighbor $)<14 \& \& q_{1}>$ rand then state $(t+1, i) \longleftarrow$ Eq;

if $\operatorname{state}(t, i)=I \& \&$ anyNeighborOfCell $(i)=H$ then state $(t+1, i) \longleftarrow H$;

if state $(t, i)=I \& \& 1 /(1+\exp ((10-$ timeLongInfected $(i)) / 2))<$ rand $\& \& q_{2}>$ rand then state $(t+1, i) \longleftarrow H$;

if $\operatorname{state}(t, i)=E \& \&$ probTurnToInfected $<$ rand \&\& probESelfHealing $\geq$ rand then $\operatorname{state}(t+1, i) \longleftarrow R$;

if state $(t, i)=I \& \& 1 /(1+\exp ((10-\operatorname{timeLongInfected}(i)) / 2))>=$ rand

\&\& $1-$ medicalLevel $<$ rand then $\operatorname{state}(t+1, i) \longleftarrow R$;

if $\operatorname{state}(t, i)=I \& \& 1 /(1+\exp ((10-\operatorname{timeLongInfected}(i)) / 2)) \geq$ rand

$\& \& 1-$ medicalLevel $\geq$ rand then state $(t+1, i) \longleftarrow D$;

if $\operatorname{state}(t, i)=\mathrm{Eq} \& \&$ probTurnToInfected $\geq \operatorname{rand}$ then $\operatorname{state}(t+1, i) \longleftarrow H$;

if $\operatorname{state}(t, i)=\mathrm{Eq} \& \&$ probTurnToInfected $<$ rand $\& \&$ probEqSelfHealing $\geq$ rand

then state $(t+1, i) \longleftarrow R$;

if state $(t, i)=H \& \& 1 /(1+\exp ((10-$ timeLongInfected $(i)) / 2)) \geq$ rand.

\&\& $1-$ medicalLevel $<$ rand then $\operatorname{state}(t+1, i) \longleftarrow R$.

if state $(t, i)=H \& \& 1 /(1+\exp ((10-$ timeLongInfected $(i)) / 2)) \geq \operatorname{rand}$

$\& \& 1-$ medicalLevel $\geq$ rand then $\operatorname{state}(t+1, i) \longleftarrow D$

//update daysAfterContact matrix and adjacency matrix

Pseudocode 1: The pseudocode of the state transformation of TS-SEIR model.

transmission, where the contact times are varied as $(3,6,9)$ and varied gathering times. In the $3^{\text {rd }}$ group, the initial infected number is varied as $(40,100,160)$ to test the effect of the infected number during the pandemic transmission but set as 100 in the other experiments. The effect of medical level to the virus control and transmission is shown in the $4^{\text {th }}$ group experiments [15].

The virus transmission is estimated via the typical SEIR models with the differential equation in which the parameters are fixed without considering the randomness of the 
virus transmission process. However, the comprehensive influencing factors are fully considered in the developed TSSEIR model. Therefore, the parameters involved in the TSSEIR model can be updated through iteration as a dynamic process for describing the gathering, medical conditions, and infected number in such varied states in the actual virus transmission situations.

\section{Simulation Results and Analysis}

3.1. Isolation Rate. It is known that isolation is the most effective measure to block the virus transmission in the absence of vaccine $[16,17]$. TS-SEIR model is used to simulate the pandemic development trend with different isolation rates (see Figure 3 ), as set in the $1^{\text {st }}$ group experiments, listed in Table 1. It can be seen from Figure 3 that the higher the isolation ratio, the lower the total number of infected individuals by quarantine during the COVID-19.

Besides, the recovery number with the highest isolation rate can also fast achieve balance compared with those of other isolation rates. For instance, the traffic restrictions were applied throughout China one week after the Wuhan City was closed down on $23^{\text {rd }}$ January 2020 , which would greatly decrease the contact possibility and scope. After a 17day buffer period, the already spread pandemic situation from $14,380\left(1^{\text {st }}\right.$ Feb 2020) to $72,436\left(17^{\text {th }}\right.$ Feb 2020) has finally been stabilized and then gradually declined, which has proved a positive effect of the high-level quarantine on the spread controlling the pandemic after the outbreak stage $\left(24^{\text {th }}\right.$ January-31 $1^{\text {st }}$ January 2020$)$.

\subsection{Infection Number prior to Policy Intervention. The} number of infection cases before intervention, which depends largely on the reaction speed and intervention measures put in place by local authorities during the initial stage of the epidemic outbreak [18], plays a crucial role in shaping the consequent infections. The development trend of pandemic under different initial infected cases $(40,60$, and 100 , respectively) is simulated for illustrative purposes. Intuitively, the more the number of people infected before the intervention, the faster the rate of early cases increased, as illustrated in Figure 4. In other words, the larger the proportion of the population infected, the more difficult the epidemic development can be contained in the latter stage. This highlights the importance of timing of initial control, i.e., early intervention measures being put in place by the health authority. More specifically, the lower the number of initial infection cases, the more likely the peak of infections may be delayed (as well as smaller magnitude of the peak); this may contribute to winning precious time for local authorities to organize and coordinate cross-sectorial actions in fighting against a virulent pandemic such as COVID-19. In short, the initial situation of the pandemic outbreak at a given region has a profound effect on the effectiveness of public health crisis management.

To illustrate the timing effect, here we compare the pandemic data of South Korea and Germany during the early stage of COVID-19 development. It is found that South
Korea had more than 200 confirmed patients when it took strict measures such as entry control on $21^{\text {st }}$ February 2020; however, the domestic infections kept increasing rapidly since then. By contrast, Germany adopted strict immigration control measures at a similar date, on $27^{\text {th }}$ February 2020 when only 40 patients were confirmed, and the consequent growth of confirmed cases in Germany was relatively slow for a certain period of time. Thus, a key takeaway is that the number of infected before the draconian pandemic prevention measures being implemented may determine the initial spread rate of the pandemic, leading to markedly different pathways of the epidemic spread and development.

3.3. Degree of Contact with Infected People. Close contact with a large number of susceptible people would highly increase the infection ratio, such as mass gathering in Church worships in Daegu of South Korea [19]. However, it is rather difficult to implement such mandatory measures in certain parts of the world.

Here, the TS-SEIR model is used to simulate the effect of the contact number on the pandemic control with a relatively high isolation rate of $0.9 / 0.99$, where the results of the development trend with different contact number are depicted in Figure 5. The number of the susceptible, exposed, and infected people with/without quarantine would be greatly reduced with less contact number. Hence, the "mobile contact network," as the mobile infection source, can greatly increase the risk/probability of being infected, especially in intensive social interconnections because of living closely to each other, i.e., India as the second largest population with confirmed infection on $8^{\text {th }}$ September 2020, reported by the WHO [2].

Another example is South Korea; strict policy had been implemented for the importation of pandemic at the early stage of the outbreak. However, domestic mobility and mass gatherings are not prohibited, such as the congregants of the Shincheonji Church of Jesus without appropriate protection measures, resulting in more than 6,000 new confirmed COVID-19 cases in two weeks, as the largest number of infections outside China at $18^{\text {th }}$ February 2020. The explosive growth of infected cases of COVID-19 in South Korea during the first week of nation's outbreak evidently demonstrates the effectiveness of noninterpersonal contact on the pandemic spread control.

In order to eliminate the risk of fast spread rate in densely populated countries in the Asia Pacific, region strict quarantine measures, i.e., prohibiting unnecessary outside activities, were forced to be implemented in Europe, like Spain and Italy, to stop the sharply interpersonal contact; otherwise, the global situation would have been lost control entirely.

3.4. Medical Capacity. It is known that the medical level plays a crucial role in managing the epidemic emergency while preventing the virus from uncontrolled spreading [20], such as the number of medical personnel involved in the treatment, the length of the confirmed diagnosis, and the number of isolation wards with negative pressure. The 


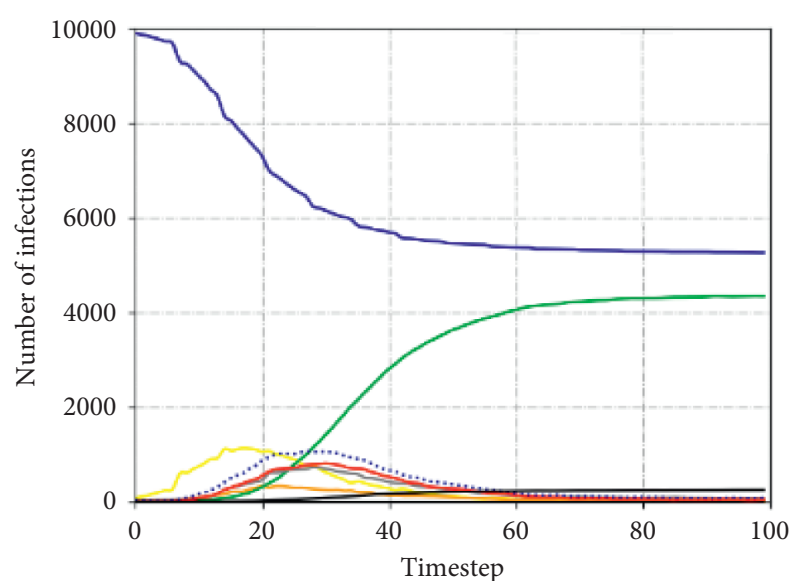

- Susceptible but not quarantined $(S)$ Exposed but not quarantined (E)

_ Infected but not quarantined $(I)$

- Recovery $(R)$

_ Susceptible and quarantined (SQ)

_ Exposed and quarantined (EQ)

_ Infected and quarantined $(H)$

.. All infected $(H+I)$

- Death $(D)$

(a)

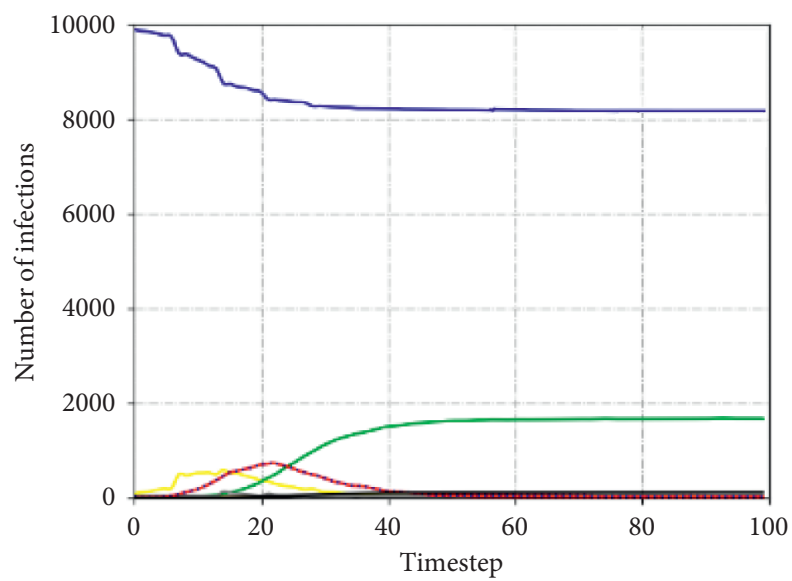

\footnotetext{
- Susceptible but not quarantined $(S)$ Exposed but not quarantined $(E)$ Infected but not quarantined $(I)$

- Recovery $(R)$

- Susceptible and quarantined (SQ)

_ Exposed and quarantined (EQ)

_ Infected and quarantined $(H)$

... All infected $(H+I)$

— Death $(D)$
}

(c)

FIGURE 3: Simulated pandemic under different isolation ratios: $q_{1} / q_{2}$ comparison analysis.

influence of the medical level on pandemic development with the TS-SEIR model is simulated under different medical levels, as shown in Figure 6. The higher the medical level index is, the lower the death rate of the patients is expected to be.

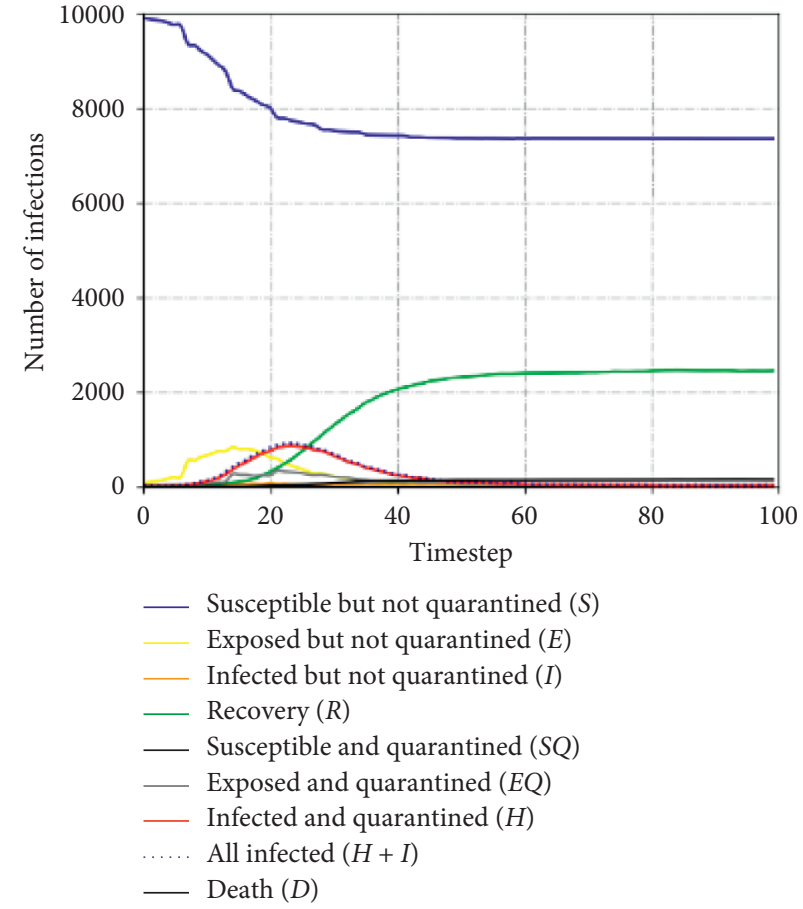

(b)

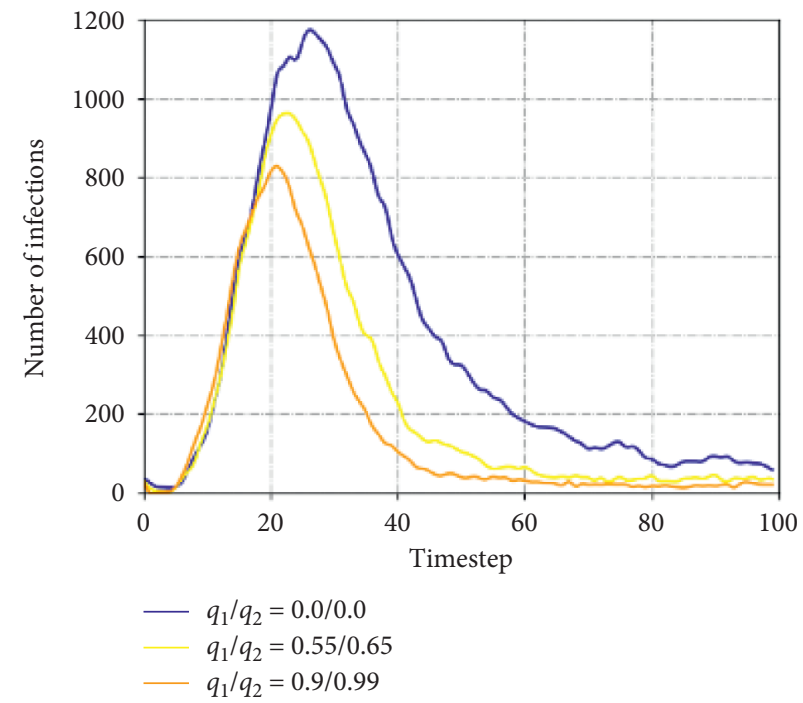

(d)

(a) $q_{1} / q_{2}=0.2 / 0.3 ;$ (b) $q_{1} / q_{2}=0.55 / 0.65 ;$ (c) $q_{1} / q_{2}=0.9 / 0.99 ;$ (d) of the epidemic, the Chinese Hubei Province, was running severely short of sufficient medical resources, and the local medical capacity could not cope with the overwhelming coronavirus outbreak, leading to a relatively high mortality 


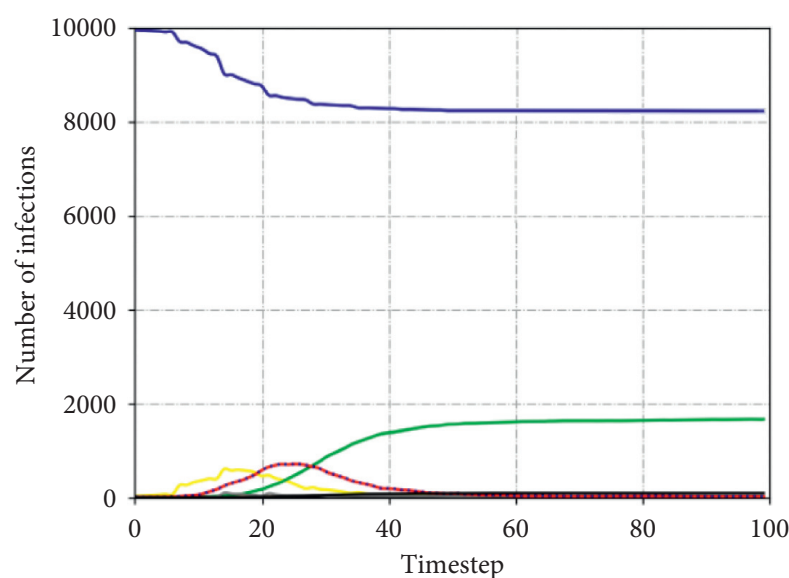

_ Susceptible but not quarantined $(S)$ Exposed but not quarantined $(E)$

_ Infected but not quarantined $(I)$

- Recovery $(R)$

_ Susceptible and quarantined (SQ)

- Exposed and quarantined (EQ)

_ Infected and quarantined $(H)$ All infected $(H+I)$

- Death $(D)$

(a)

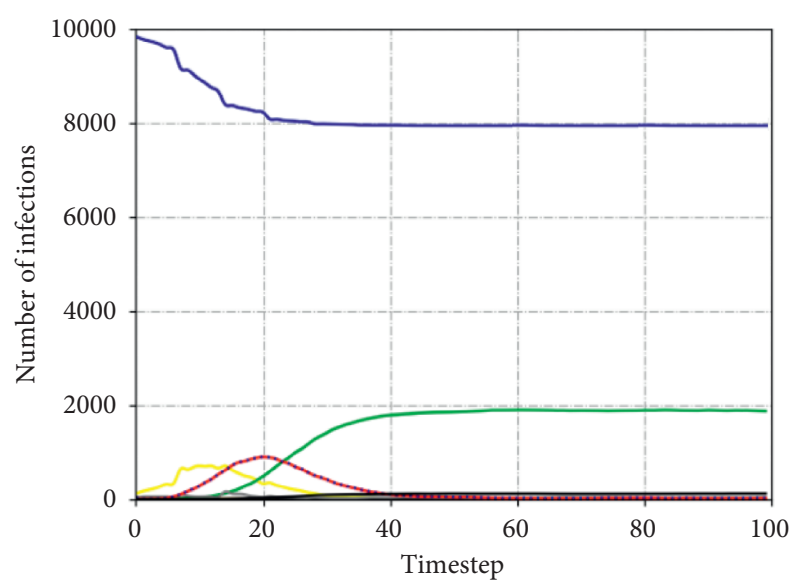

\footnotetext{
Exposed but not quarantined $(E)$

Infected but not quarantined $(I)$

Recovery $(R)$

- Susceptible and quarantined (SQ)

- Exposed and quarantined (EQ)

- Infected and quarantined $(H)$

. All infected $(H+I)$

Death $(D)$
}

- Susceptible but not quarantined $(S)$

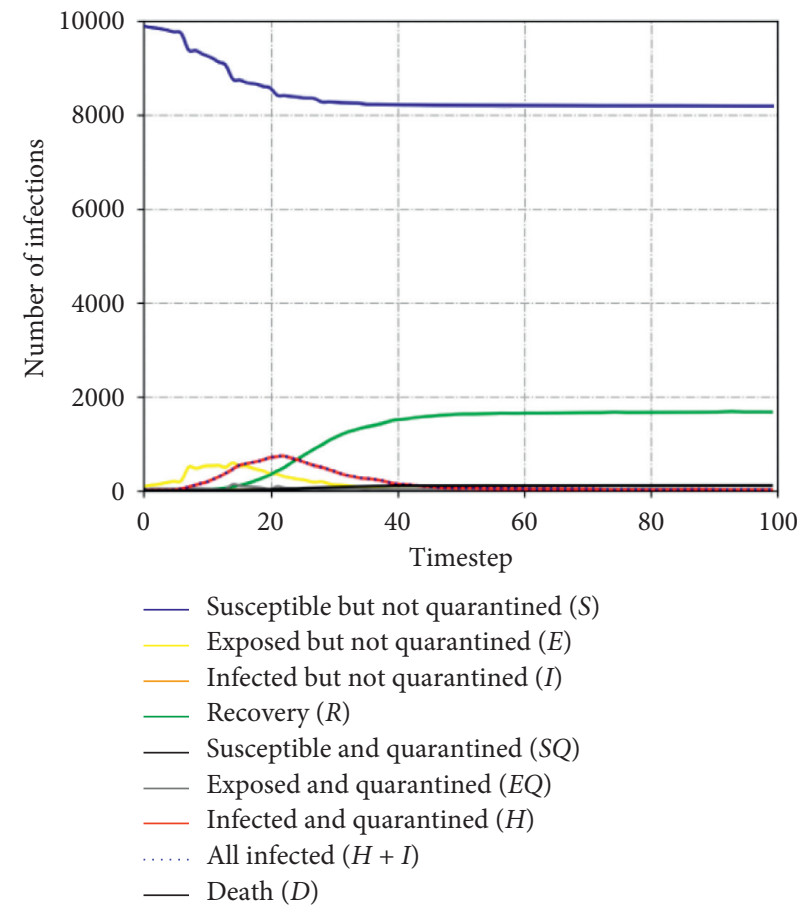

(b)

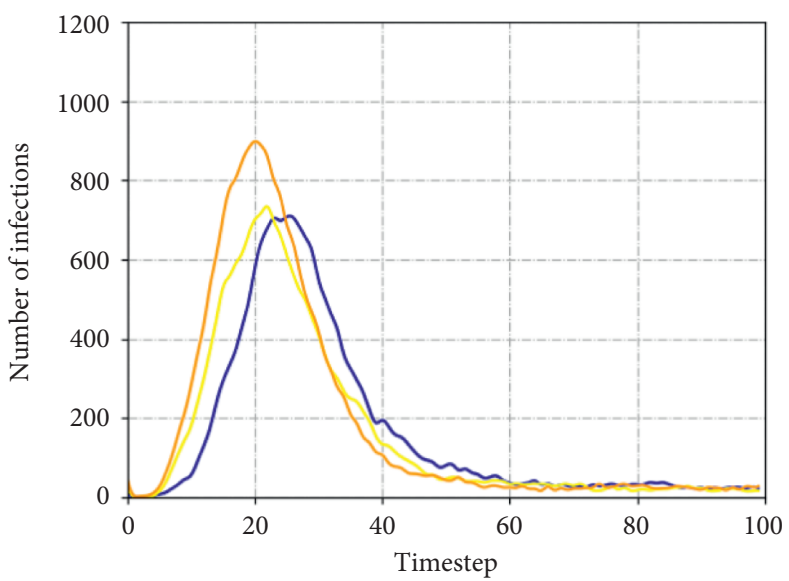

- Initial infections $=40$

Initial infections $=100$

Initial infections $=160$

(c)

(d)

FIGURE 4: Simulated pandemic under different initial infected numbers: (a) initial infections=40; (b) initial infections=100; $(c)$ initial infections $=160$; (d) initial infections comparison analysis

rate during the initial period of pandemic development. The Chinese government realised the serious medical challenge and immediately commanded building makeshift hospitals which began receiving patients on $8^{\text {th }}$ February 2020, and the nationwide medical forces were rapidly mobilised and dispatched to assist with the saturated medical staff in Hubei Province so that the medical resources of Hubei Province were replenished and the treatment of patients soon returned to adequate level. From $8^{\text {th }}$ February 2020, the number of deaths per day started to slowly increase and 


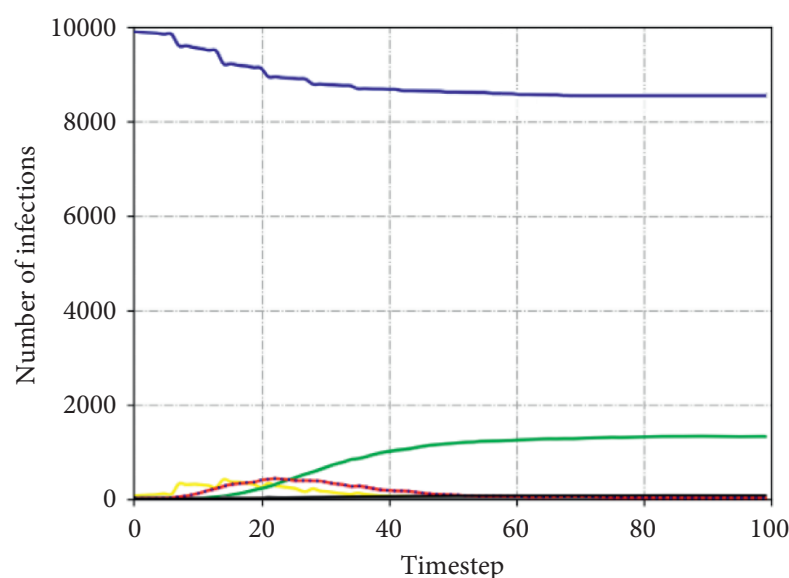

- Susceptible but not quarantined $(S)$ Exposed but not quarantined $(E)$

- Infected but not quarantined $(I)$

- Recovery $(R)$

- Susceptible and quarantined (SQ)

_ Exposed and quarantined (EQ)

- Infected and quarantined $(H)$ All infected $(H+I)$

— Death $(D)$

(a)

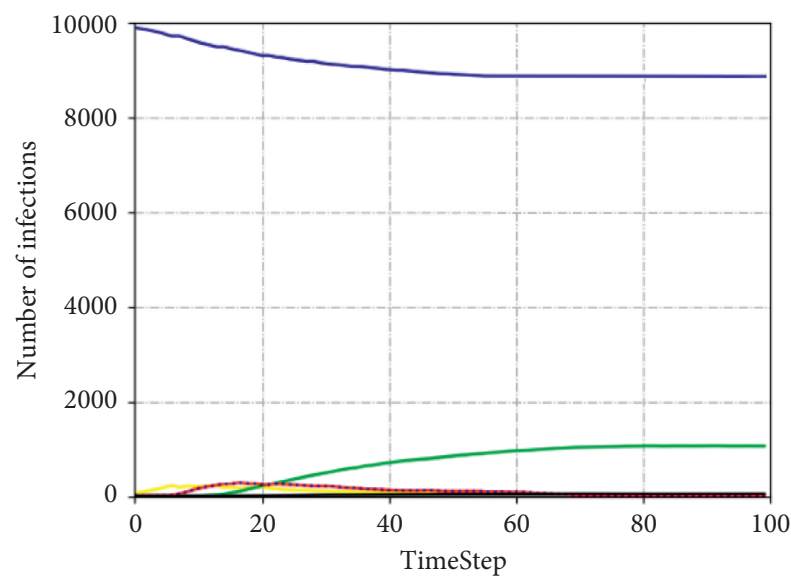

_ Susceptible but not quarantined $(S)$

Exposed but not quarantined $(E)$

Infected but not quarantined (I)

- Recovery $(R)$

_ Susceptible and quarantined (SQ)

_ Exposed and quarantined (EQ)

_ Infected and quarantined $(H)$

All infected $(H+I)$

- Death $(D)$

(c)

Figure 5: Simulated pandemic under different contact numbers: (a) $\mathrm{Ct} / \mathrm{Gt}=3 / 80 *($ susceptible/all); (b) $\mathrm{Ct} / \mathrm{Gt}=6 / 80 *($ susceptible/all);

(c) $\mathrm{Ct} / \mathrm{Gt}=6 / 0 *($ susceptible/all); (d) $\mathrm{Ct} / \mathrm{Gt}$ comparison analysis.

gradually declined, thanks to the improved medical level, which fully reflected the impact of medical conditions on the mortality rate associated with the pandemic.

The parameter-wise adjustment simulation experiment results clearly demonstrate that the restrained interpersonal contact or interactions are probably the most effective

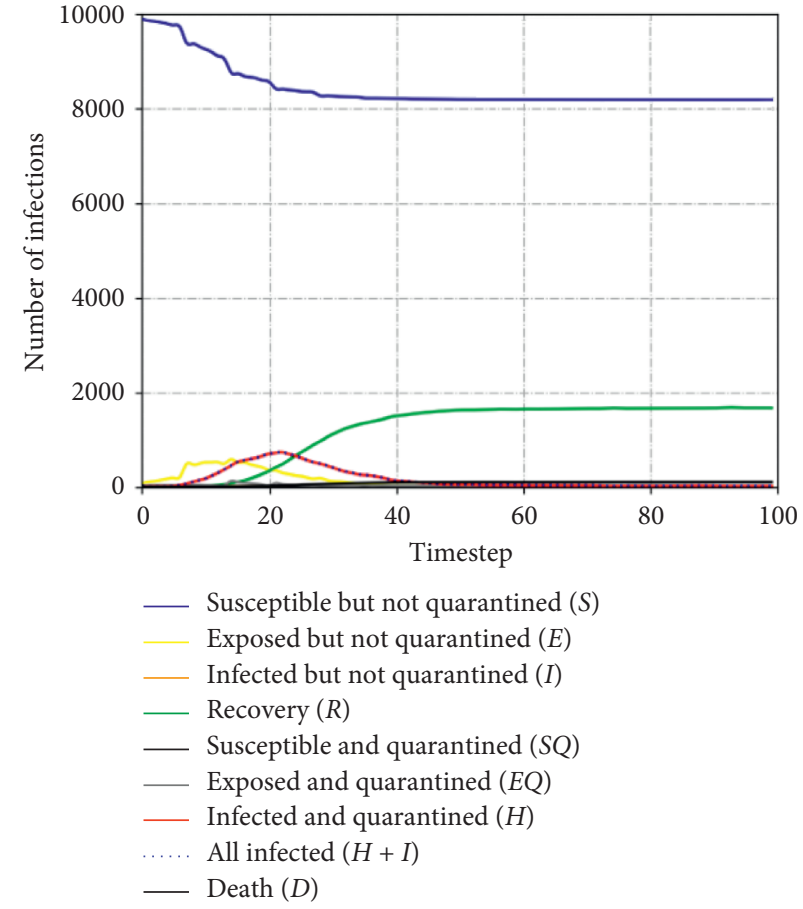

(b)
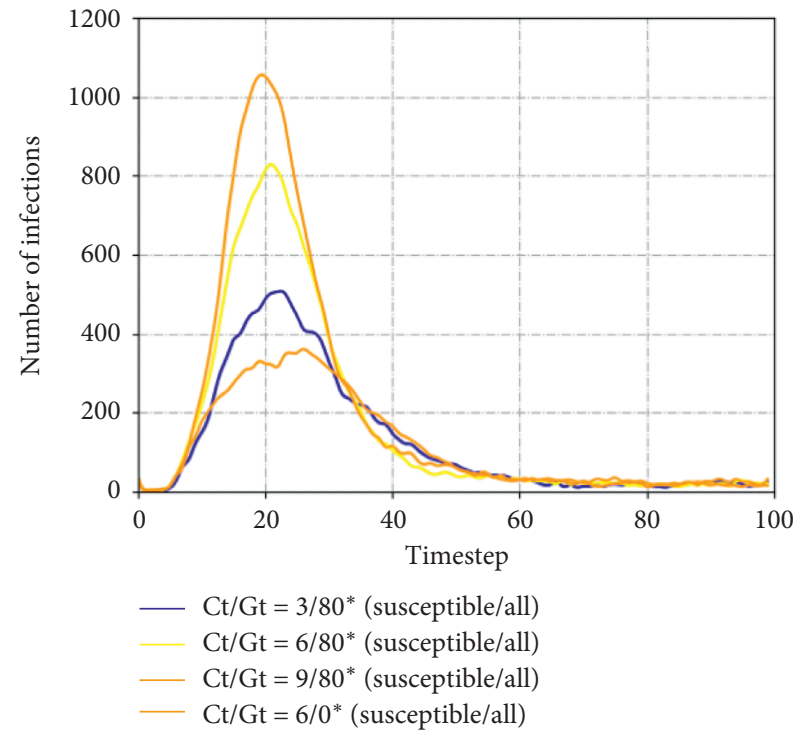

(d) strategy to mitigate the spread of the virus along with sufficient medical treatment to cut off the transmission route and protect the population from the susceptible and the infected [16]. To summarize, a key lesson of the TS-SEIR model suggests that the proactive measures such as prevention and control prior to large-scale epidemic outbreak 


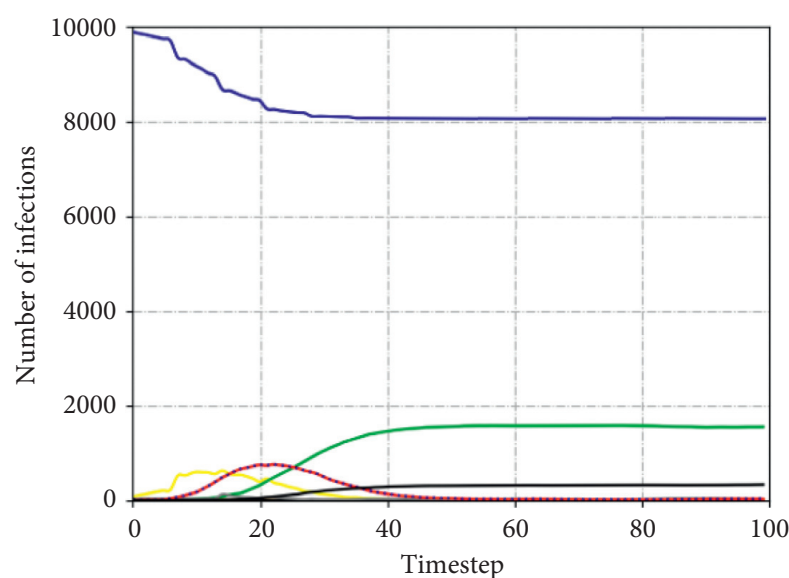

- Susceptible but not quarantined $(S)$ Exposed but not quarantined $(E)$

Infected but not quarantined $(I)$

- Recovery $(R)$

- Susceptible and quarantined $(S Q)$

Exposed and quarantined $(E Q)$

— Infected and quarantined $(H)$ . All infected $(H+I)$

— Death $(D)$

(a)

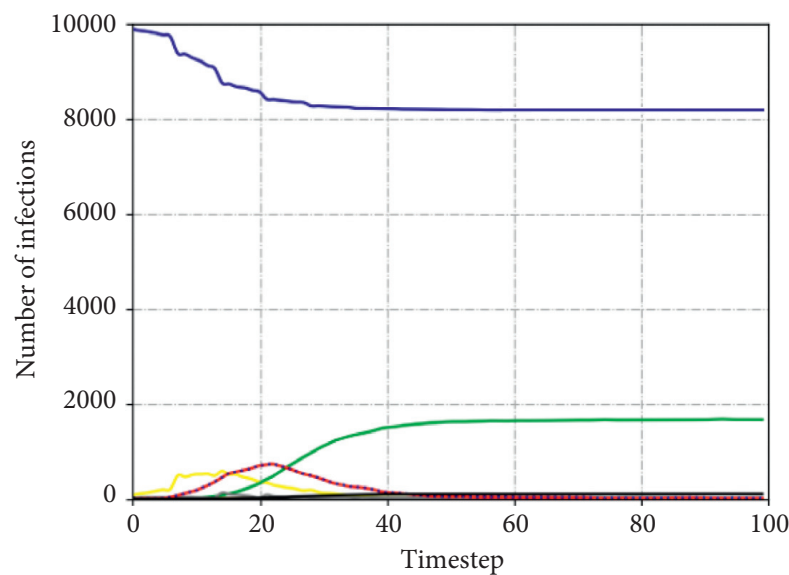

- Susceptible but not quarantined $(S)$

Exposed but not quarantined $(E)$

Infected but not quarantined $(I)$

- Recovery $(R)$

- Susceptible and quarantined (SQ)

— Exposed and quarantined $(E Q)$

— Infected and quarantined $(H)$

..... All infected $(H+I)$

_ Death $(D)$

(c)

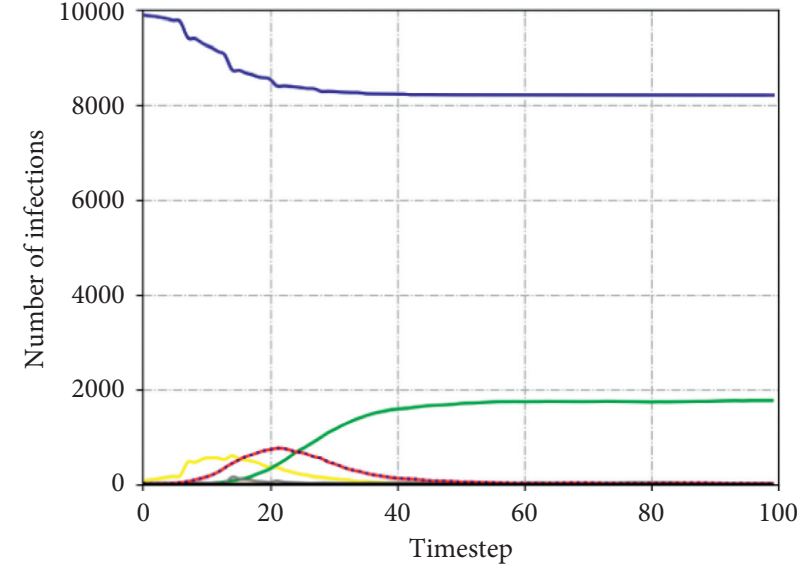

- Susceptible but not quarantined $(S)$

Exposed but not quarantined $(E)$

— Infected but not quarantined $(I)$

— Recovery $(R)$

- Susceptible and quarantined (SQ)

— Exposed and quarantined $(E Q)$

— Infected and quarantined $(H)$

..... All infected $(H+I)$

— Death $(D)$

(b)

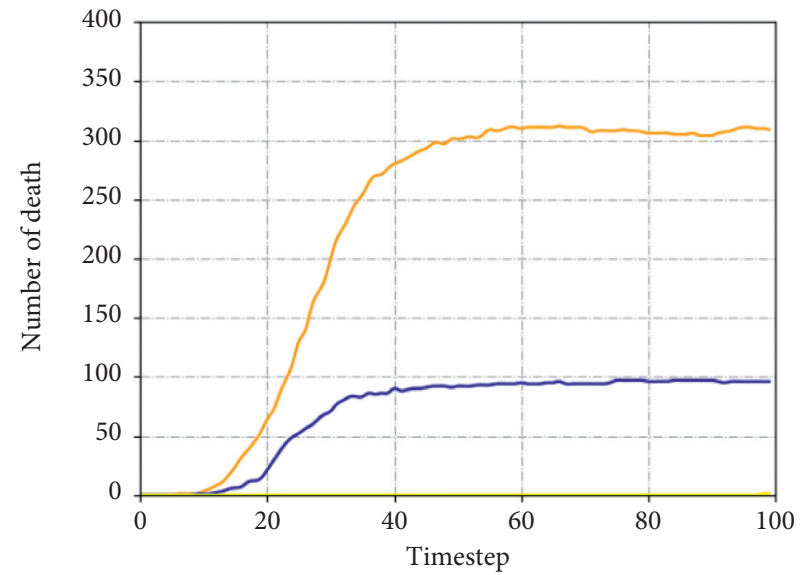

Medical level $=0.8293$

— Medical level $=0.9455$

Medical level $=0.9994$

(d)

Figure 6: Simulated pandemic under different medical levels: (a) medical level=0.8293; (b) medical level=0.9994; (c) medical lev$\mathrm{el}=0.9455$; (d) medical level comparison analysis.

are more important than the medical countermeasures including diagnostics and clinical treatment for containing the spread of a virulent pandemic such as COVID-19 because the vaccines are still under development and specific cure is not yet available [15].

\section{Discussion of the COVID-19 Situation}

In the aspect of proactive pandemic management, emergency response should be launched in a timely manner in conjunction with preemptive alarm response and a priori 
TABLE 1: The involved parameter setting of the TS-SEIR model for pandemic development simulation.

\begin{tabular}{|c|c|c|c|c|c|}
\hline No. & Isolation rate $q_{1} / q_{2}$ & $\begin{array}{l}\text { Normal contact } \\
\text { times }\end{array}$ & $\begin{array}{c}\text { Gathering contact } \\
\text { times }\end{array}$ & $\begin{array}{l}\text { Initial number of } \\
\text { infected }\end{array}$ & Medical level index \\
\hline Group 1 & $\begin{array}{c}(0.2 / 0.3,0.55 / 0.65,0.9 / \\
0.99)\end{array}$ & 6 & $80 \times($ Suscep/all $)$ & 100 & 0.9455 \\
\hline Group 2 & $0.9 / 0.99$ & $(3,6,9,6)$ & $\begin{array}{c}80 \times(\text { Suscep/all }) \\
80 \times(\text { Suscep/all }) \\
80 \times(\text { Suscep/all }) \\
0 \times(\text { Suscep/all })\end{array}$ & 100 & 0.9455 \\
\hline Group 3 & $0.9 / 0.99$ & 6 & $80 \times($ Suscep/all $)$ & $(40,100,160)$ & 0.9455 \\
\hline Group 4 & $0.9 / 0.99$ & 6 & $80 \times($ Suscep/all $)$ & 100 & $\begin{array}{c}(0.9455,0.9994 \\
0.8293)\end{array}$ \\
\hline
\end{tabular}

Note: Suscep $=$ susceptible.

risk assessment system. The primary objective of the preventive intervention measures is to cut off the route of transmission and to protect the susceptible population by eliminating the source of infections based on the integrated approach, such as reducing public gatherings and close interpersonal contact, good hygiene practice (wearing masks outdoors and washing hands frequently), suspending social and religious gathering activities, limiting traffic and mobility, strengthening quarantine at urban and intercity transportation hubs, closing temporarily access to educational institutions and public places, and carrying out thorough disinfection wherever necessary [21]. In addition, increasing the efficiency of isolation of infected population, early diagnosis, and treatment are highly recommended, including routine temperature check of vulnerable population, screening, and monitoring of symptom-susceptible patients and centralised isolation of the suspected cases and confirmed patients.

In the event of high risk of contamination, draconian quarantine measures such as home confinement of the entire infected areas with tight mobility restriction should be implemented (such as the lockdown policies adopted by a number of European countries to prohibit all nonessential outdoor activities) [22]. Both our modelling results and the experience of the Chinese city of Wuhan (one of the main reasons of the high mortality rate of the infected patients in Wuhan during the early stage of the outbreak of COVID-19) were related to the saturation in local medical resources including overwhelmed treatment centres and lack of caring personnel. The situation only started to improve after the Chinese central government sent more than 50,000 medical staff to the city and more than 15 makeshift hospitals started operation to admit tens of thousands of patients with mild symptoms of novel coronavirus for the purpose of isolation. Thus, the treatment capacity is a determining factor in reducing the mortality of infected patients. This invaluable experience gained from China's pandemic combat may be shared with the countries facing similar challenges), suggesting that all nonnecessary outdoor activities, in particular, the social gatherings, and mobility should be suspended immediately to minimise the interpersonal transmission likelihood. Also, nationwide medical resources have to be mobilised timely to provide necessary medical support to the most infected areas to gain the struggle with the virus transmission. Furthermore, temporary medical isolation centres may be built to increase the patient intake capacity in the event of serious outbreak.

Another controversial issue is related to the prevention and control measure (P\&CM) options with regard to the tradeoff between proactive and laissez-faire attitude. Indeed some European countries (e.g., UK and Sweden) had initially tempted the so-called nonpharmaceutical interventions (NPIs), or herd immunity strategy faced with rapidly increased infection cases and high pressure on local medical services (e.g., hospitalisation infrastructures to intake patients with critical situation), due to the fact that the local treatment capacities had been quickly saturated as a consequence of unpreparedness and lack of coordination of social resources while the optimal timing of strong intervention was missed out; however, the ultimate outcome of this passive tackling largely relying on the NPIs finally proved disastrous, and policymakers had to make a policy $U$ turn by re-enforcing proactive pandemic control measures to prevent the health crisis from deteriorating. This is in line with our modelling results which clearly stressed the importance of initial actions in retarding the peak of infections, which is crucial to managing the long-term epidemic spread as a whole.

The success of joint implementation of the pandemic control guideline depends upon the response of emergency plan such as cutting off the route of transmission and protecting the susceptible population with isolation and treatment of infectious diseases [22]. An integrated resources management and allocation system needs to be established to allow local medical and social workers to jointly complete the work of screening and controlling the sources of infection.

With the rapid spread of novel coronavirus worldwide, this pandemic was no longer a single country's affair but has been developing into a global pandemic requiring cooperation and control in all countries [23]. The pandemic has so far spread across over 210 countries in the world. In many countries and regions, effective prevention and control are still "new topic" that has not been addressed in the previous pandemic outbreaks such as H1N1 or Ebola virus. The pandemic has not been fully brought under control even in many developed countries such as the United States, where the total infections exceeded 6 million with hundreds of thousands of infected 
people perished their lives due to ineffective control measures during the initial stage of the outbreak. In Europe, the situations in countries such as United Kingdom, Italy, Germany, France, and Sweden are also concerning, and each of them has recorded more than 250,000 confirmed cases. Based on our modelling analysis of the impact of aggregation factors on the development of the pandemic, it is likely to expect a second-wave outbreak across European and the Middle East countries, should no radical health policy be undertaken by the national governments in due course.

Another critical issue in terms of lifecycle management of pandemic is related to the precautionary measures to prevent a potential second-wave outbreak after the previously infected region or country has reached the stabilised state; e.g., all domestic infectious origins have been cleared off. In other words, how to minimise the risk of importing new cases for the disinfected origin of outbreak poses a serious challenge for local public health. This is particularly relevant to the current situations in East Asian countries with a large degree of increased mobility and high risk of exposure to international travels, which is facing a high risk of imported cases. Any mishandling or improper prevention of the imported cases, a secondary transmission of novel coronavirus, might lead to the outbreak anew, and it is noteworthy that a reinfection case has been recently confirmed in Hong Kong (on $1^{\text {st }}$ March 2020, one imported case from UK has been found in Shenzhen; on $2^{\text {nd }}$ March 2020, Qingtian County, Zhejiang Province, had 7 imported confirmed cases from Italy; before that, Beijing and Ningxia had 3 imported confirmed cases from Iran; these figures seem to be small compared with the overall scale of the existing confirmed cases in China, while they bring new challenges to the pandemic prevention and control in China) [24].

Last but not the least, the transparent and fluent information flow plays a critical role in coordinating pandemic control measures across different stakeholders, especially on social network platforms which may contribute to relaying and processing pandemic risk-related information through the whole process of pandemic control campaign for the well-being of international community. On the one hand, the timely release of information on the outbreak of highly contagious epidemic in an initially infected area is conducive to the prevention and control of infectious diseases in other countries and regions, which is instrumental to the global governance of a COVID-19-alike pandemic. Also, nonbiased and transparent disease-related intelligence information can help scientific understanding of new infectious diseases and pandemic situation of the public and stabilizing social mood, on the other hand. How to prevent the psychological panic from spreading across mass population is essential for health authorities when dealing with an emergent public health crisis with an unprecedented scale such as COVID-19. To gain legitimacy and public trust in local authority and health care institutions, the golden triangle of punctuality, accuracy, and transparency of pandemic-related information should be consistently maintained at all times.

\section{Conclusion}

The global experience of fighting against the COVID-19 pandemic across various countries clearly indicates that the efficient management of the person-to-person transmission risk of the epidemic requires taking timely preventive measures in global and national public health system, which is in line with our hybrid modelling results. In particular, serious consequences would be generated if the optimal window of intervention were forfeited at the early stage of outbreak, in particular, in the densely populated areas (i.e., the intranetwork transmission probability may be increased exponentially) with scarce medical resources.

The most effective P\&CM globally of such infectious diseases as novel coronavirus mainly includes enhancing proactive pandemic management to maximising the efficiency of integrated public health intervention measures [25]. During the initial period of P\&CM implementation, quantitative analysis can be carried out with a SEIR-type model introduced in the present study, i.e., TS-SEIR model, by focusing on key control parameters such as the estimated transmission rate, evaluating the possible impact of the pandemic according to the preliminary epidemiological investigation, and predicting the required resources, scale, and time to formulate and implement the intervention plan aided by medical experts and public health policymakers.

The interdisciplinary and intergovernmental coordination is also a prerequisite of the success in combatting the global public health crisis. The shared knowledge of pandemic control parameters may provide useful lessons for countries on the set of increasing risks of large infections. As such, it is an imperative for decision makers from international and national institutions to undertake efficient countermeasures based on robust modelling of the likelihood of pandemic spread pathways, whereby the frontier between policy arena and academia should be connected in an collaborative and open spirit for preserving the global public goods of human health and social welfare.

\section{Data Availability}

All data used to support the findings of the study are available from the corresponding author upon reasonable request.

\section{Conflicts of Interest}

The authors declare that there are no conflicts of interest.

\section{Authors' Contributions}

JL and YMZ conceived and designed the study. ZGC, QSL, and LJY set up the experiments. XDW, HYN, and CNF collected the data. ZGC, HYN, and CNF analysed the data. JL, YMZ, ZGC, and LJY interpreted the results and wrote the manuscript. Yimin Zhou, Jun Li and Lingjian Ye are contributed equally. 


\section{Acknowledgments}

This work was partially supported by the National Science Foundation of China (Grant no. 61973296).

\section{References}

[1] B. Chen, E. K. Tian, B. He et al., "Overview of lethal human coronaviruses," Signal Transduction and Targeted Therapy, vol. 5, no. 1, pp. 1-16, 2020.

[2] World Health Organization (WHO), "Coronavirus disease 2019 (COVID-19) situation report-48," 2020, https://covid19. who.int/WHO-COVID-19-global-data.csv.

[3] T. P. B. Thu, P. N. H. Ngoc, and N. M. Hai, "Effect of the social distancing measures on the spread of COVID-19 in 10 highly infected countries," Science of the Total Environment, vol. 742, Article ID 140430, 2020.

[4] C. H. Watts, P. Vallance, and C. J. M. Whitty, "Coronavirus: global solutions to prevent a pandemic," Nature, vol. 578, no. 7795 , p. $363,2020$.

[5] C. P. Novel, "The epidemiological characteristics of an outbreak of 2019 novel coronavirus diseases (COVID-19) in China," Zhonghua Liu Xing Bing Xue Za Zhi, vol. 41, no. 2, pp. 145-151, 2020.

[6] A. C. K. Lee, N. A. Alwan, and J. R. Morling, "Wuhan novel coronavirus (COVID-19): why global control is challenging?" Public Health, vol. 179, pp. A1-A2, 2020.

[7] K. Kupferschmidt and J. Cohen, "Can China's COVID-19 strategy work elsewhere?” Science, vol. 367, no. 6482, pp. 1061-1062, 2020.

[8] B. F. Maier and D. Brockmann, "Effective containment explains subexponential growth in recent confirmed COVID-19 cases in China," Science, vol. 368, no. 6492, pp. 742-746, 2020.

[9] Y. Fang, Y. Nie, and M. Penny, "Transmission dynamics of the COVID-19 outbreak and effectiveness of government interventions: a data-driven analysis," Journal of Medical Virology, vol. 92, no. 6, pp. 645-659, 2020.

[10] J. T. Wu, K. Leung, and G. M. Leung, "Nowcasting and forecasting the potential domestic and international spread of the 2019-nCoV outbreak originating in Wuhan, China: a modelling study," The Lancet, vol. 395, no. 10225, pp. 689-697, 2020.

[11] L. Tang, Y. Zhou, L. Wang et al., "A review of multi-compartment infectious disease models," International Statistical Review, vol. 88, no. 2, pp. 462-513, 2020.

[12] G. Giordano, F. Blanchini, R. Bruno et al., "Modelling the COVID-19 epidemic and implementation of population-wide interventions in Italy," Nature Medicine, vol. 26, no. 6, pp. 855-860, 2020.

[13] S. Ghosh and S. Bhattacharya, "A data-driven understanding of COVID-19 dynamics using sequential genetic algorithm based probabilistic cellular automata," Applied Soft Computing, vol. 96, Article ID 106692, 2020.

[14] A. Teslya, T. M. Pham, N. G. Godijk et al., "Impact of selfimposed prevention measures and short-term government intervention on mitigating and delaying a COVID-19 epidemic," PLoS Med, vol. 17, no. 7, Article ID e1003166, 2020.

[15] L. Li, Z. Yang, Z. Dang et al., "Propagation analysis and prediction of the COVID-19," Infectious Disease Modelling, vol. 5, pp. 282-292, 2020.

[16] B. Nussbaumer-Streit, V. Mayr, A. I. Dobrescu et al., "Quarantine alone or in combination with other public health measures to control COVID-19: a rapid review," Cochrane
Database Systematic Review, vol. 4, no. 4, pp. 501-506, Article ID CD013574, 2020.

[17] T. Basso, S. A. Nordbø, E. Sundqvist, T. C. Martinsen, E. Witsø, and T. S. Wik, "Transmission of infection from nonisolated patients with COVID-19 to health care workers," Journal of Hospital Infection, vol. S0195-6701, no. 20, pp. 30402-30403, 2020.

[18] S. Zhao and H. Chen, "Modeling the epidemic dynamics and control of COVID-19 outbreak in China," Quantitative Biology (Beijing, China), vol. 8, pp. 11-19, 2020.

[19] S. Choi and M. Ki, "Estimating the reproductive number and the outbreak size of COVID-19 in Korea," Epidemiology and Health, vol. 42, Article ID e2020011, 2020.

[20] M. Salathé, C. L. Althaus, R. Neher et al., "COVID-19 epidemic in Switzerland: on the importance of testing, contact tracing and isolation," Swiss Medical Weekly, vol. 150, Article ID w20225, 2020.

[21] D. D. Rajgor, M. H. Lee, S. Archuleta, N. Bagdasarian, and S. C. Quek, "The many estimates of the COVID-19 case fatality rate," The Lancet Infectious Diseases, vol. 20, no. 7, pp. 776-777, 2020.

[22] H. Sjödin, A. Wilder-Smith, S. Osman, Z. Farooq, and J. Rocklöv, "Only strict quarantine measures can curb the coronavirus disease (COVID-19) outbreak in Italy, 2020," Euro Surveillance, vol. 25, no. 13, Article ID 2000280, 2020.

[23] A. Pan, L. Liu, C. Wang et al., "Association of public health interventions with the epidemiology of the COVID-19 outbreak in Wuhan, China," JAMA, vol. 323, no. 19, pp. 1915-1923, 2020.

[24] H. Ledford, "Coronavirus reinfections: three questions scientists are asking," Nature, vol. 585, no. 7824, pp. 168-169, 2020.

[25] K. Prem, Y. Liu, T. W. Russell et al., "The effect of control strategies to reduce social mixing on outcomes of the COVID19 epidemic in Wuhan, China: a modelling study," The Lancet Public Health, vol. 5, no. 5, pp. e261-e270, 2020. 